\title{
Perioperative subepicardial aneurysm (impending myocardial rupture) after drug eluting stent thrombosis
}

\author{
Edward Kosik, DO • Yamini S. Levitzky, MD • \\ Charles E. Smith, MD · Robert S. Finkelhor, MD
}

Received: 13 January 2009/Revised: 20 January 2009/ Accepted: 20 January 2009/Published online: 14 February 2009

(c) Canadian Anesthesiologists' Society 2009

\section{To the Editor,}

Drug eluting stents (DES) are impregnated with cytostatic compounds to reduce the restenosis rate. Unfortunately, these cytostatic compounds also inhibit normal endothelial growth, which is accompanied by an increased risk of thrombogenesis. We report a case with catastrophic outcome involving a patient with several previously placed DES who was undergoing cancer surgery.

The patient, a 71-year-old male, was scheduled for resection of a rectal carcinoma 6 months after DES placement. He had been diagnosed with an ischemic cardiomyopathy (LV ejection fraction 37\%) for which three DES and three bare metal stents had been placed.

Five days prior to the patient's surgery, clopidogrel and aspirin were held, and heparin was used as a "bridge". On the day of his surgery, the partial thromboplastin time was $121 \mathrm{sec}$, the prothrombin time was $14.4 \mathrm{sec}$, and the international normalized ratio (INR) was 1.3. Metoprolol, lisinopril, nitroglycerin, and lorazepam were continued. General anesthesia was induced with etomidate and fentanyl, and isoflurane, remifentanil, and metoprolol were used during maintenance. Anesthesia and surgery were uneventful; duration was $4.5 \mathrm{hr}$. The patient's blood loss

E. Kosik, DO · C. E. Smith, MD (ه)

Department of Anesthesia, MetroHealth Medical Center,

Case Western Reserve University School of Medicine,

Cleveland, $\mathrm{OH}$, USA

e-mail: csmith@metrohealth.org

Y. S. Levitzky, MD - R. S. Finkelhor, MD

Heart and Vascular Center, MetroHealth Medical Center,

Case Western Reserve University School of Medicine,

Cleveland, $\mathrm{OH}$, USA was $200 \mathrm{~mL}$; his urine output was $150 \mathrm{~mL}$, and $3000 \mathrm{~mL}$ of lactated Ringer's solution was infused. At the completion of the case, the patient's trachea was extubated, and he was transferred to the ICU.

Immediately postoperatively, large ST elevations were noted in the anterior ECG leads. Multiple vasopressors (norepinephrine, epinephrine, and phenylephrine) were infused, and the patient's trachea was reintubated. Twelve hours later, when anticoagulation was considered to be acceptable, cardiac catheterization was carried out, and percutaneous coronary intervention (PCI) of a completely thrombosed DES in the mid-left anterior descending artery was performed. Despite an intra-aortic balloon pump and supportive therapy, the patient developed cardiogenic shock. Thirty hours after the infarction, a transthoracic echocardiogram showed worsened left ventricular function (LVEF 20\%) with a jagged echolucency at the apex that filled with left heart echocardiographic contrast consistent with a subepicardial aneurysm (impending rupture, Fig. 1). Medical care was withdrawn on postoperative day five.

The risk of stent thrombosis with aspirin and clopidogrel administration is $0.4 \%$. If antiplatelets are discontinued prematurely, the risk of thrombosis increases dramatically. ${ }^{1}$ The risk of bleeding in patients taking dual antiplatelets may be less dangerous than previously thought and depends on the type of surgery and other factors. ${ }^{2}$ In 2007, a science advisory from the American Heart Association, the American College of Cardiology, the Society for Cardiovascular Angiography and Interventions, the American College of Surgeons, and the American Dental Association recommended the following: to continue dual antiplatelets for a period of $1 \mathrm{yr}$ after DES placement, to continue aspirin indefinitely, to delay non-cardiac surgery for 6 weeks after PCI with bare metal stent, and to delay noncardiac surgery for 12 months after PCI with DES. ${ }^{3}$ 
Fig. 1 Oblique apical transthoracic echocardiographic views showing a subepicardial aneurysm (arrows) both with and without echocardiographic left heart contrast

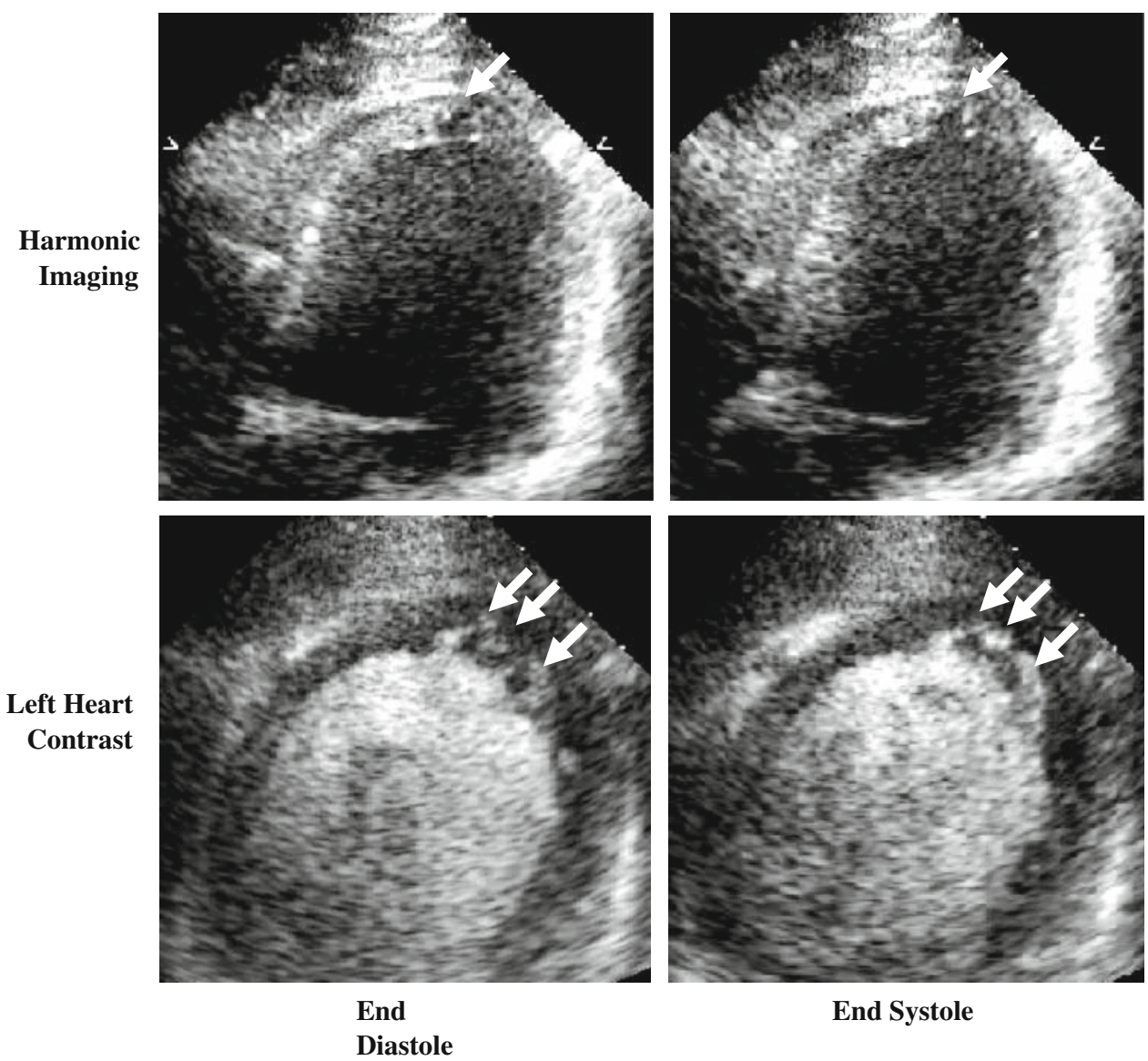

Ventricular rupture is a recognized complication of an ST-elevation myocardial infarction. However, finding an incomplete myocardial tear prior to rupture (subepicardial aneurysm) is a rare but important finding, as early surgical intervention could be lifesaving. ${ }^{4}$ Recognizing this pathology is even more relevant, since late stent thrombosis is a major concern in DES patients discontinuing antiplatelet medication. ${ }^{2,5}$ Little information exists on the development of a subepicardial aneurysm after a DES thrombosis. However, it is expected that this complication will be encountered more often with the rise in DES use, with the likely need for subsequent non-cardiac surgery in a growing elderly population, and with unproven protection techniques for perioperative stent thrombosis. ${ }^{2,3}$ Thus, recognizing its echocardiographic appearance is even more important. Further, the use of echocardiographic left heart contrast may enhance the early recognition of this entity. It is possible that, as this phenomenon becomes better appreciated, not all patients will suffer a myocardial rupture; some may develop a true aneurysm and/or a markedly trabeculated akinetic infarction.
Conflicts of interest None declared.

\section{References}

1. Vicenzi MN, Meislitzer T, Heitzinger B, Halaj M, Fleisher LA, Metzler $H$. Coronary artery stenting and non-cardiac surgery-a prospective outcome study. Br J Anaesth 2006; 96: 686-93.

2. Charbucinska KN, Godet $G$, Itani $O$, et al. Anticoagulation management for patients with drug-eluting stents undergoing vascular surgery. Anesth Analg 2006; 103: 261-3.

3. Grines $C L$, Bonow RO, Casey DE Jr, et al. Prevention of premature discontinuation of dual antiplatelet therapy in patients with coronary artery stents: a science advisory from the American Heart Association, American College of Cardiology, Society for Cardiovascular Angiography and Interventions, American College of Surgeons, and American Dental Association, with representation from the American College of Physicians. J Am Coll Cardiol 2007; 49: 734-9.

4. Giltner A, Marelli D, Halpern E, Savage M. Subepicardial aneurysm with impending cardiac rupture: a case of antemortem diagnosis and review of the literature. Clin Cardiol 2007; 30: 44-7.

5. Dala $A R$, D'Souza $S$, Shulman $M S$. Brief review: coronary drugeluting stents and anesthesia. Can J Anesth 2006; 53: 1230-43. 